\title{
Reaction Enhancement of Initially Distant Scalars by Lagrangian Coherent Structures.
}

\author{
Kenneth R. Pratt, ${ }^{1,}$ a) John P. Crimaldi, ${ }^{1, b)}$ and James D. Meiss ${ }^{2, c)}$ \\ 1) Department of Civil, Environmental and Architectural Engineering \\ University of Colorado, Boulder, Colorado 80309-0428 USA \\ 2) Department of Applied Mathematics \\ University of Colorado, Boulder, Colorado 80309-0526 USA
}

(Dated: 22 August 2014)

Turbulent fluid flows have long been recognized as a superior means of diluting initial concentrations of scalars due to rapid stirring. Conversely, experiments have shown that the structures responsible for this rapid dilution can also aggregate initially distant reactive scalars and thereby greatly enhance reaction rates. Indeed, chaotic flows not only enhance dilution by shearing and stretching, but also organize initially distant scalars along transiently attracting regions in the flow. To show the robustness of this phenomenon, a hierarchical set of three numerical flows is used: the periodic wake downstream of a stationary cylinder, a chaotic double gyre flow, and a chaotic, aperiodic flow consisting of interacting Taylor vortices. We demonstrate that Lagrangian coherent structures, as identified by ridges in finite time Lyapunov exponents, are directly responsible for this coalescence of reactive scalar filaments. When highly concentrated filaments coalesce, reaction rates can be orders of magnitude greater than would be predicted in a well-mixed system. This is further supported by an idealized, analytical model that was developed to quantify the competing effects of scalar dilution and coalescence. Chaotic flows, known for their ability to efficiently dilute scalars, therefore have the competing effect of organizing initially distant scalars along the LCS at timescales shorter than that required for dilution, resulting in reaction enhancement.

PACS numbers: 47.27.De,47.51.+a,47.70.Fw

\footnotetext{
a)Electronic mail: kenneth.pratt@colorado.edu

b)Electronic mail: john.crimaldi@colorado.edu
} 
${ }^{c}$ Electronic mail: james.meiss@colorado.edu 


\section{INTRODUCTION}

The stirring and subsequent mixing of passive scalars in turbulent or chaotic flows is of vital importance for a variety of natural and anthropogenic systems. Whether it is the stirring of plankton on the free surface of the ocean, ${ }^{1}$ or the mixing of reactive gases

in a combustion chamber, ${ }^{2}$ turbulent stirring plays a significant role in the transport and dispersion of scalars. Chaotic dynamics provides a general framework to study the advection of scalars and the theory of mixing, areas that had previously been studied on a case-bycase basis. ${ }^{3}$ The fundamental processes responsible for the complexity of chaotic advection are the repeated stretching and folding of fluid elements. Stretching leads to exponential divergence of nearby fluid parcels (evidenced by positive Lyapunov exponents), while folding gives rise to chaotic stirring. The resulting large concentration gradients of the advected scalars then leads to enhanced diffusive mixing.

Chaotic stirring has been studied extensively as an efficient means of quickly diluting concentrated scalars. ${ }^{3,4}$ A blob of dye, for example, will quickly be stretched into thin filaments as it is advected in a chaotic flow, leading to an exponential decay of concentration with time. Though many studies have focused on the mixing of single scalars, few have looked at the complex interactions that can occur for a pair of reactive species in a turbulent/chaotic environment. Most of the latter studies focus on initial conditions where the reactive scalars share an interface. ${ }^{5,6}$ In this case, molecular diffusion results in immediate onset of reaction between the scalars and advection leads to increasing reaction rates as the interface between scalars is lengthened and sharpened by stretching. In many natural systems, however, reactive scalars do not initially share an interface, and it is fluid stirring that initiates a reaction. ${ }^{7,8}$ In this topology, the initial reaction rate is zero, and stirring leads to reaction only if it causes the initially segregated scalars to come into contact.

\section{A. Enhanced Reaction of Initially Distant Scalars}

Both numerical ${ }^{9,10}$ and experimental ${ }^{11}$ studies have shown that the stirring of initially distant scalars may result in their eventual overlap. When the concentration of the filaments is not too low, these coalescence events have been shown to lead to reaction rates much greater than would be predicted by a well-mixed system, a process that we refer to as 
reaction enhancement. Crimaldi et al. ${ }^{9}$ considered a simple point vortex and showed-both numerically and analytically - that the vortical strain can result in reaction rates greatly exceeding that of pure diffusion. Next, Crimaldi and Kawakami ${ }^{10}$ studied coalescence events downstream of a stationary cylinder that shed periodic vortices. Here, the strain between the counter-rotating downstream vortices resulted in the coalescence of filaments that were initially separated upstream of the cylinder. The coalescence of concentrated filaments is due to the structured stirring from a superposition of vortices, which creates regions of coherent strain and results in the stretching and coalescence of nearby filaments. The mechanisms that lead to this coalescence, however, have not yet been studied. In the current paper, we will elaborate on these mechanisms and quantify the resulting enhanced reaction rates.

\section{B. Lagrangian Coherent Structures}

The visualization of coherent structures within turbulent flows dates back to the time of Leonardo da Vinci. ${ }^{12}$ In flowing water, da Vinci saw eddies of many different sizes that interacted with each other in a complex manner, a prelude to the work of Richardson and the energy cascade. The coherent structures, in this view, were Eulerian in nature: turbulent structure was viewed as an instantaneous snapshot of a scalar field that highlights persistent, coherent regions. A component of vorticity, for example, was often used to track regions of persistent rotation, which can then be characterized as coherent. More recently, the Lagrangian viewpoint has become a useful tool to uncover coherent structures that persist across space and time. By adopting a frame of reference that moves with fluid parcels, we gain a natural means of describing the transport of passive scalars in fluids that have a complicated time history of stretching and folding. ${ }^{13}$

Lagrangian coherent structures (LCS) have been defined as material lines or surfaces in fluid flows that either enhance or inhibit mixing. ${ }^{14}$ These time-evolving surfaces have been described as the hidden skeleton of a flow because of how they mold the complex patterns

of a passive tracer. ${ }^{15,16}$ Haller showed that such material surfaces could be either attracting or repelling, analogous to the invariant unstable and stable manifolds of hyperbolic orbits in time-periodic chaotic systems. ${ }^{13}$ Fluid parcels in the neighborhood of such surfaces are either attracted or repelled at an exponential rate. Repelling LCS (rLCS) act as transport barriers in fluid flows. These have been studied, for example, in a geophysical context to time 
the release of pollutants near a coastline in order to make them predominantly wash out to sea. ${ }^{17}$ By contrast, attracting LCS (aLCS), analogous to unstable manifolds, are attracting surfaces for a passive scalar. For example, larvae on the ocean surface have been shown to aggregate from different source regions onto an aLCS. ${ }^{18}$ Not surprisingly, marine predators have also been shown to track aLCS in the ocean in order to locate food patches. ${ }^{19}$

One standard technique to locate the repelling and attracting surfaces in fluid flows, is the Finite-Time Lyapunov Exponent (FTLE). The FTLE is a scalar field that can be estimated by seeding the domain with a uniform grid of tracer particles and advecting these particles either forward or backward in time. After an integration time $T$, the rate at which neighboring particles have separated is calculated for each grid point, as described in Shadden et al. ${ }^{20}$ Forward integration yields the repelling surfaces by finding the local maximum rate of separation, while backward integration gives attracting surfaces by finding the maximum rate of contraction. The integration time - for example a typical turnover time - must be selected by the physics of the flow to determine features of interest. Lagrangian coherent structures can be extracted from the FTLE field by locating its ridge lines. It is important to note, however, that ridges in an FTLE field can erroneously identify regions of high shear that are not true attracting or repelling LCS. $^{15}$

\section{METHODOLOGY}

Reactions between initially distant scalars form through some combination of stirring at large scales and diffusion at small scales. We consider reactions that obey second order kinetics, with the reaction rate given by

$$
R(x, t)=k C_{1}(x, t) C_{2}(x, t)
$$

where $k$ is the reaction rate constant, and $C_{1}$ and $C_{2}$ are the concentrations of the two scalars. The location and magnitude of the resulting reactions depends on the details of the flow field. In this paper, we demonstrate that Lagrangian coherent structures provide a template for locations where reactions are likely to occur. To demonstrate the generality of the correspondence between LCS and reaction zones, we will study three models. Each has a 2D incompressible flow and is dominated by vortex interactions.

The first flow is the periodic vortex wake behind a stationary cylinder, the second is the time-periodic double gyre, and the third consists of an interacting set of Taylor vortices. 
This last flow is both aperiodic and chaotic, and is most representative of a $2 \mathrm{D}$ turbulent velocity field.

\section{A. Flow Field Descriptions}

\section{Cylinder Wake}

The first flow is the laminar cylinder wake from Crimaldi and Kawakami ${ }^{10}$ where a uniform flow with speed $u_{0}$ impinges on a circular cylinder with diameter $a$. When $R e=$ $u_{0} a / \nu=100$, this leads to the periodic shedding of vortices with a period $T_{0}=9.3$, downstream of the cylinder. Representative streamlines for this flow are shown in Fig. 1. In order to separate the underlying vortex motion from the ambient flow (Fig. 1a), streamlines are also computed for $\mathbf{u}=\left(u-0.95 u_{0}, v\right)$ (Fig. 1b). Immediately downstream of the cylinder, counter-rotating vortices are periodically shed leading to localized Lagrangian chaos. ${ }^{21}$ The separatrix region between these vortices is an area of significant fluid straining. ${ }^{22}$ Particle paths further downstream are not chaotic; nevertheless, the separatrices persist and are asymptotic to stable and unstable manifolds of a steady flow.

A pair of reactive scalars (red and blue) with constant concentration over a width $w$ and lateral separation $s$, are continuously released upstream of the cylinder at $x=-2.4 a$ (as

shown in Fig. 6). The scalars obey an advection-diffusion equation with diffusivity $D$. The velocity and scalar fields were computed numerically using a finite-element discretization of the Navier-Stokes and reactive transport equations within the COMSOL Multiphysics package. Details are given by Crimaldi and Kawakami. ${ }^{10}$

\section{Double Gyre}

The second flow is a simplified dynamical model of a double gyre (Fig. 2), an incompressible flow consisting of two counter-rotating vortices that alternately expand and contract periodically. The flow exhibits Lagrangian chaos and has been used in many previous studies as a test-bed for Lagrangian diagnostics. ${ }^{20,23}$ The velocity field is given by

$$
\begin{aligned}
& u=-\pi U \sin (\pi f(x)) \cos (\pi y), \\
& v=\pi U \cos (\pi f(x)) \sin (\pi y) \frac{\partial f}{\partial x},
\end{aligned}
$$


a)

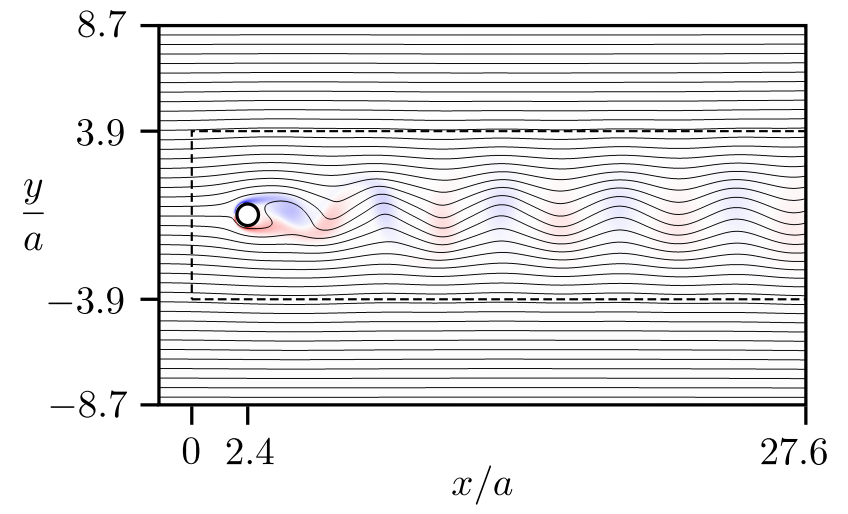

b)

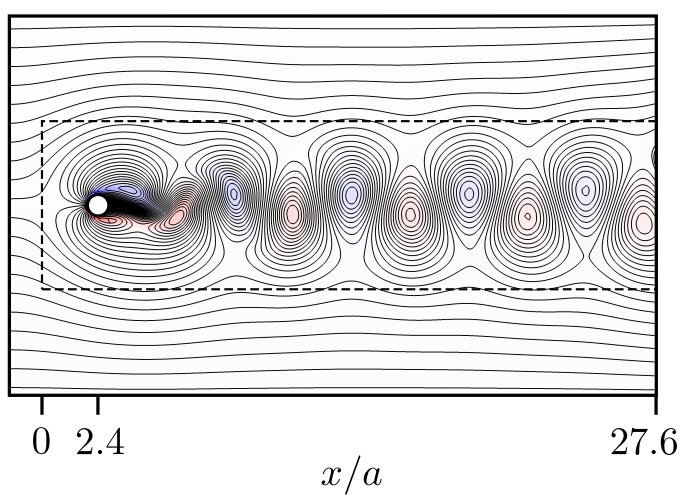

FIG. 1. a) Streamlines $\mathbf{u}=(u, v)$ and vorticity for the cylinder wake at $\operatorname{Re}=100$. b) In order to separate the underlying vortex motion from the ambient flow, the streamlines are computed for $\mathbf{u}=\left(u-0.95 u_{0}, v\right)$. Regions of positive (red) and negative (blue) vorticity are superimposed on the streamlines. The dashed box in a) and b) denotes the cropped subregion shown in Figs. 5 and 6.

where

$$
f(x, t)=x\left[1+\epsilon \sin \left(2 \pi t / T_{0}\right)(x-2)\right] .
$$

For the computations below, we choose velocity magnitude $U=0.1$, perturbation amplitude $\epsilon=0.25$, and oscillation period $T_{0}=10$. Scalar concentrations are computed using a Lagrangian particle tracking method as discussed in Crimaldi et al. ${ }^{9}$ and are advected using a second-order improved Euler scheme.

\section{Interacting Taylor Vortices}

The third example, in the tradition of Aref, ${ }^{24}$ consists of a collection of interacting Taylor vortices; it serves as a simple model for $2 \mathrm{D}$ incompressible turbulence. From a dynamical systems perspective, the system of interacting Taylor vortices is the most complex used in this study, as it is both aperiodic and chaotic. A Taylor vortex centered at the origin has angular velocity

$$
u_{\theta}=U r \exp \left(-\frac{r^{2}}{2 r_{0}^{2}}\right)
$$



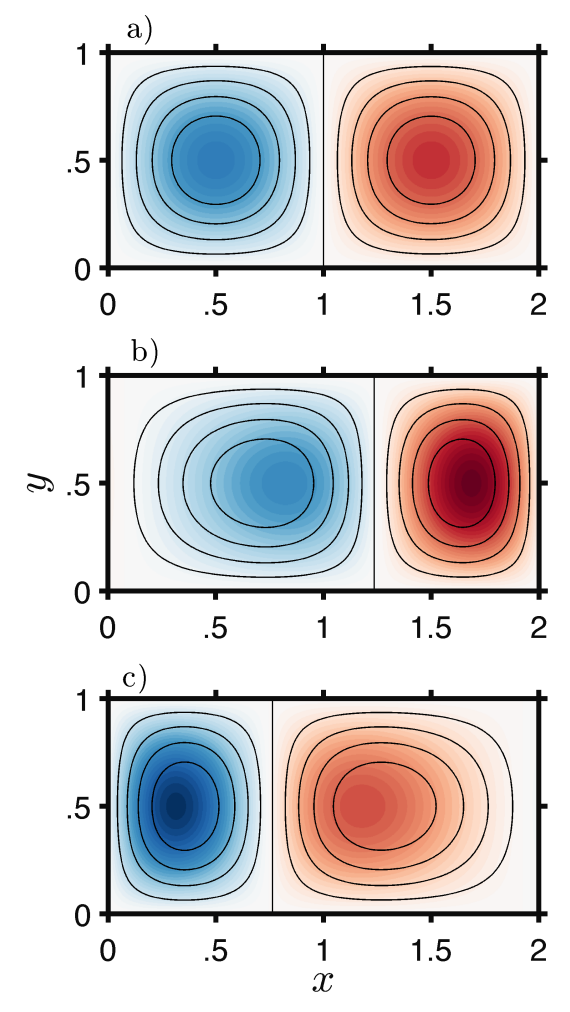

FIG. 2. Streamlines and vorticity (red is positive and blue is negative) for the double-gyre flow at quarter-period intervals. a) $t=0, T_{0} / 2, T_{0}$. b) $t=T_{0} / 4$. c) $t=3 T_{0} / 4$.

where $U$ is the strength of the vortex, $r$ is the distance from its center, and $r_{0}$ is the radius of the core. This is one member of a family of exact vortex solutions to the Navier-Stokes equations. ${ }^{25}$ This shielded vortex, shown in Fig. 3, has a relatively compact rotational core which then transitions to a nearly irrotational, sheared outer region. In our model, the center of each Taylor vortex is advected by a superposition of the velocities from the remaining vortices, but each vortex has fixed shape and strength. The vortices move in a square with domain size $10 r_{0}$, and periodic boundary conditions are imposed. For this model, 36 vortices are placed randomly in the domain, as shown in Fig. 4a; each vortex has radius $r_{0}=1$ and strength $U= \pm 0.5$. The sign of $U$ determines the rotation direction-clockwise or counterclockwise - and it is chosen randomly; in our simulation there were 14 positive and 22 negative vortices. The superposed velocity field of the vortices at $t=0$, as well as a contour plot of the total vorticity, is shown in Fig. 4b. Vortices are advected (by the superposed velocity at the vortex center) using a second-order improved Euler scheme. 


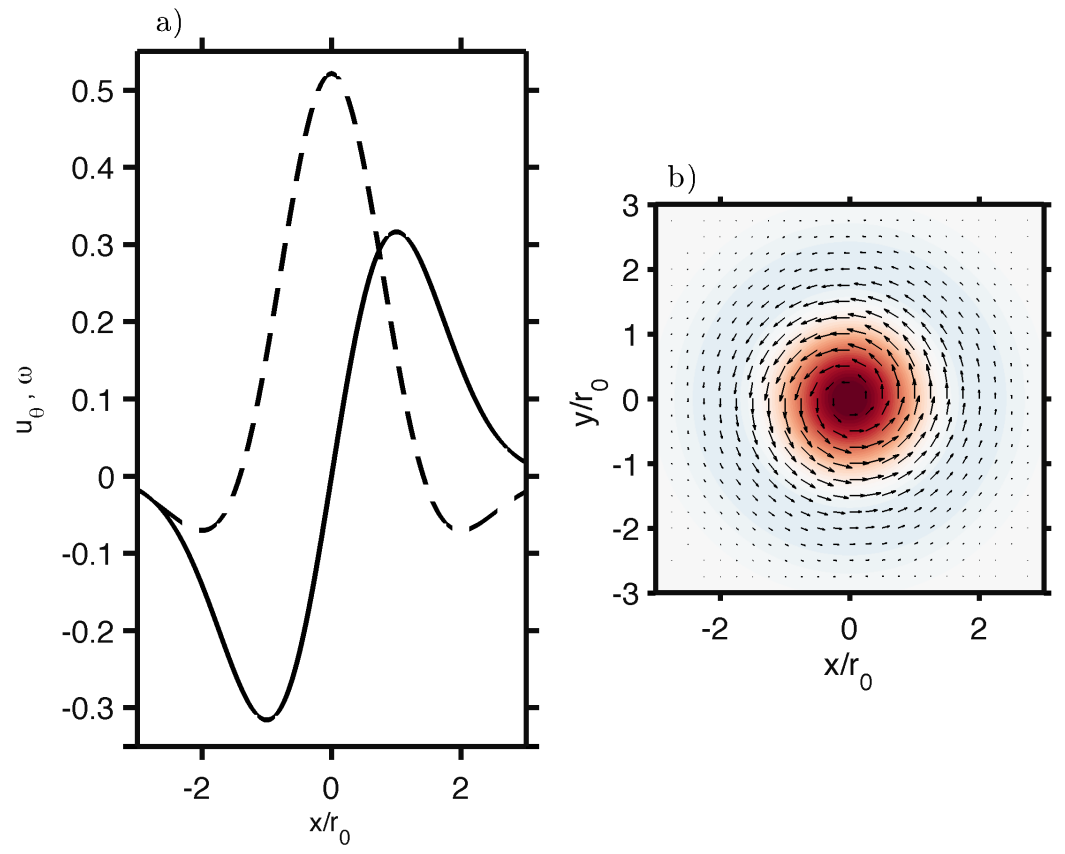

FIG. 3. Single Taylor vortex: a) velocity (solid line) and vorticity (dashed line) on a slice through the core; b) velocity vectors overlaid onto the vorticity field (colors).

Scalar concentrations are computed in the same manner as the double gyre.

\section{B. Calculating Finite Time Lyapunov Exponents}

Backward finite-time Lyapunov exponents were computed for the three flows following the method of Shadden et al. ${ }^{20} \mathrm{~A}$ flow map $\phi_{t_{0}}^{t_{0}-T}$ is generated by advecting an initially uniform array of points $(350 \times 1200$ for the cylinder flow, $201 \times 401$ for the double gyre, and $401 \times$ 401 for the Taylor vortices) backwards from $t=t_{0}$ over an integration time $T$. In this study, $T$ ranged between 2 and 2.5 periods or turnover times of the flow in order to resolve the appropriate dynamics (for a discussion of the appropriate choice for $T$ in reacting flows, see App. A). Approximate derivatives of $\phi_{t_{0}}^{t_{0}-T}$ with respect to initial conditions are computed using centered finite differences to generate the so-called right Cauchy-Green deformation tensor

$$
\Delta=\frac{d \phi_{t_{0}}^{t_{0}-T}(\mathbf{x})^{*}}{d \mathbf{x}} \frac{d \phi_{t_{0}}^{t_{0}-T}(\mathbf{x})}{d \mathbf{x}}
$$



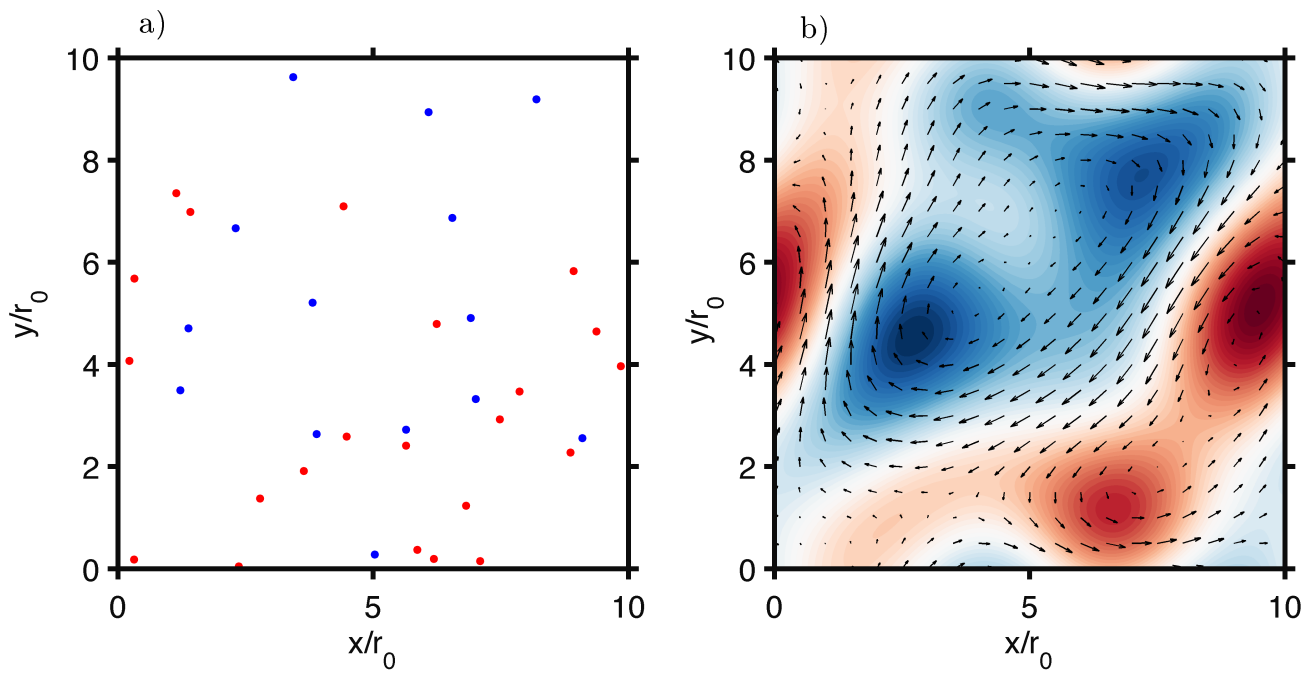

FIG. 4. a) Initial locations and direction of rotation (red is positive, blue negative) for the 36 vortices used in the Taylor vortex flow. b) Velocity field and vorticity for the superposition of the Taylor vortices in (a).

Finally, the FTLE is computed as

$$
\sigma_{t_{0}}^{T}(\mathbf{x})=\frac{1}{2 T} \ln \left(\lambda_{\max }(\Delta)\right)
$$

where $\lambda_{\max }(\Delta)$ is the maximum eigenvalue of $\Delta$. The topological ridge lines of the spatial distribution of the FTLE field correspond to aLCS, a region of strain which attracts nearby particle trajectories.

\section{RESULTS}

\section{A. LCS as a Template for Reaction}

\section{Cylinder Wake}

The FTLE field for the periodic cylinder wake computed using Eq. (2) is shown in Fig. 5. Since the far downstream wake is not chaotic, increasing $T$ does not grow the manifolds as it would for chaotic systems, but it does sharpen the ridges.

Spatial distributions of scalar concentrations and corresponding reaction rates for the cylinder wake are shown for three different scalar releases in Fig. 6. The left column shows 


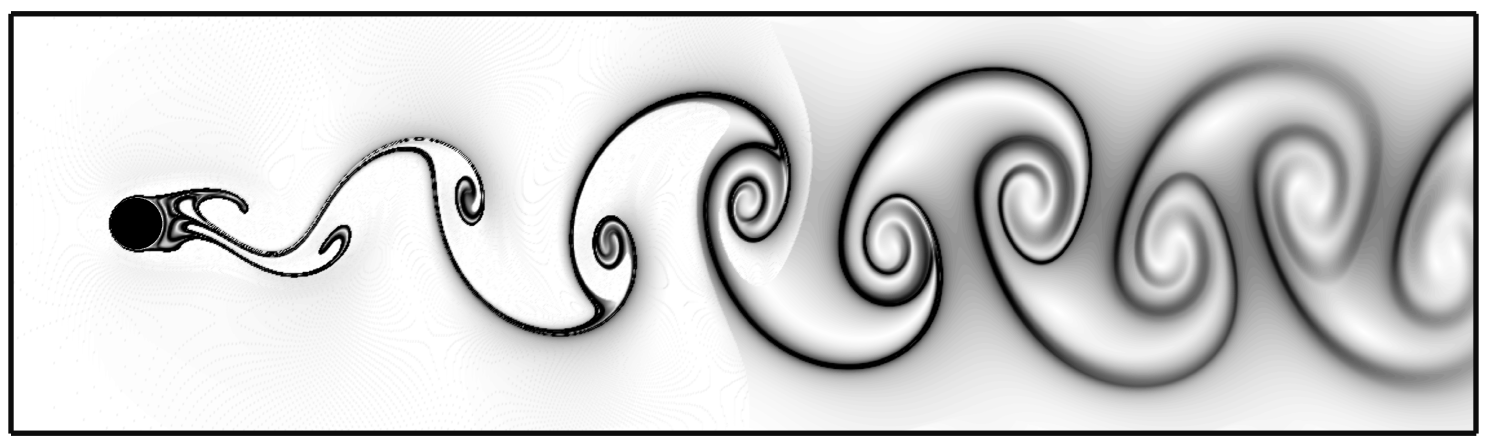

FIG. 5. FTLE field at a single phase of the cylinder wake for $T=25$, approximately 2.5 times the shedding period, $T_{0}=9.3$.

concentrations of $C_{1}$ (blue) and $C_{2}$ (red) for three sets of parameters at $t=80$. The two scalars are initially separated upstream of the cylinder and they begin coalescing in the wake as stirring and diffusion act to bridge the initial separation. The right column shows resulting reaction rates $R$ (purple) from Eq. (1), superimposed on the FTLE field for the same flow phase (gray).

Note that though the FTLE field is solely a function of the flow (and integration time $T$ ), the spatial distribution of the concentration and reaction rates depends upon both the flow and the details of the scalar diffusivity and release positions. Nonetheless, the FTLE ridges serve as a template for the reaction locations. Though the presence of an LCS does not guarantee that there will be a local reaction, where there is a reaction, it invariably corresponds to a ridge. The larger initial scalar separation in Fig. 6(a) delays the onset of reaction until further downstream relative to (b), for which initial separation is smaller. As a consequence, there are locations near the cylinder for case (a) where there is no reaction on the LCS, while for case (b) the reaction migrates upstream along the LCS. These two cases also have different scalar diffusivities (as quantified by the Schmidt number $S c=\nu / D)$. Reaction filaments always have finite widths, whereas the LCS is, in principle, simply a curve. When the diffusivity is larger, as in case (a), the reaction filament is wider and extends further spanwise from the centerline of the LCS. For the set of parameters, Fig. 6(c), the scalars are both released to one side of the cylinder; this demonstrates that even asymmetric scalar release conditions result in reaction zones that are constrained to the ridges of the FTLE. 

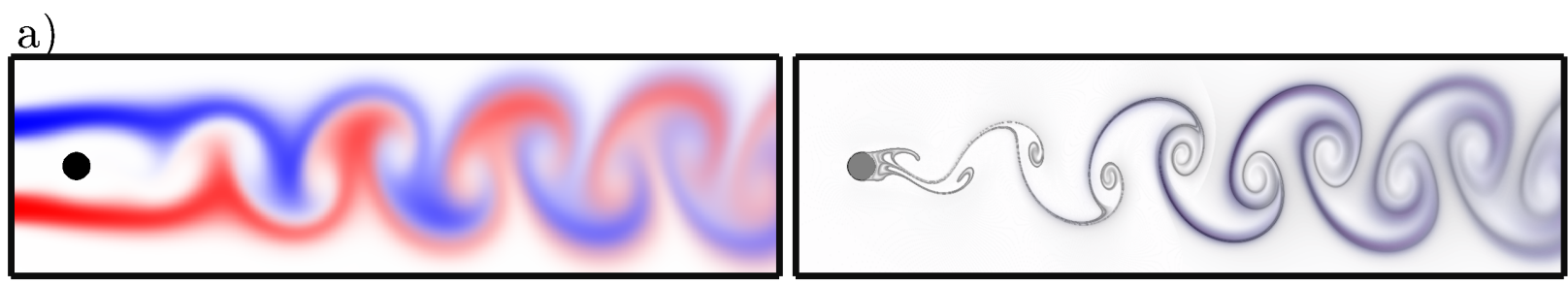

b)
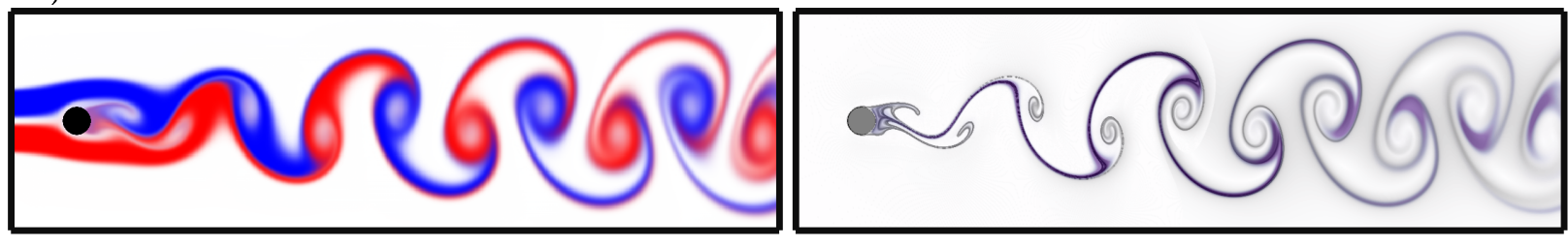

C)
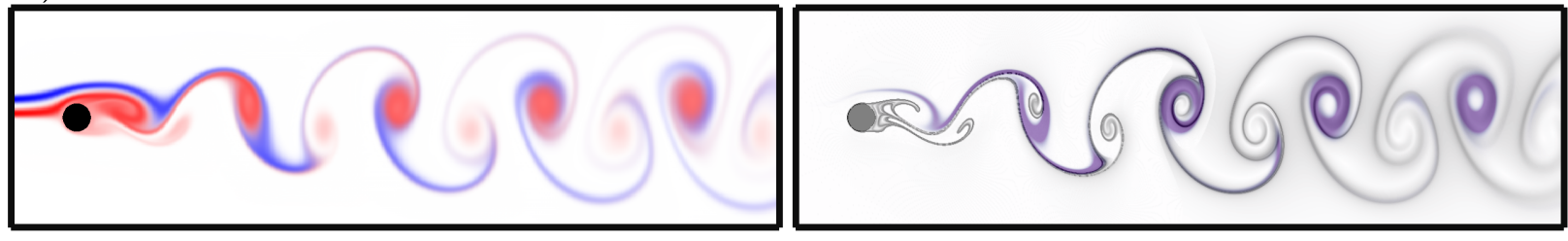

FIG. 6. The left column shows scalar concentrations (red and blue) and the right shows reaction rates (purple) superimposed on the corresponding FTLE field (gray) for the cylinder wake. a) $s=2 a, w=a$, and Schmidt number $S c=1$. b) $s=0.25 a, w=a$, and $S c=10$. c) An asymmetric scalar release with $s=0.25 a, w=a$, and $S c=10$.

\section{Double Gyre}

Figure 7 shows the evolution of the scalar concentrations and corresponding reaction rates for the double gyre flow for four sequential times. As in Fig. 6, the left column shows scalar concentrations, and the right column shows corresponding reaction rates superimposed on the FTLE field. The first row shows the initial conditions; because the scalars are separated, there is no reaction. As time evolves, scalars begin coalescing and reacting on the LCS. The figures in the second row shows that by $t=2.5 T_{0}$, multiple red and blue filaments have been attracted to the same manifold and that reaction has begun near the unstable periodic orbit on the upper boundary. Continual stretching along the unstable manifold of this saddle brings these filaments into closer contact, further enhancing the reaction, as shown in the third and fourth rows. As in the previous case, reactions do not occur everywhere on an LCS ridge, but where reactions do occur, they are centered on these ridges. 

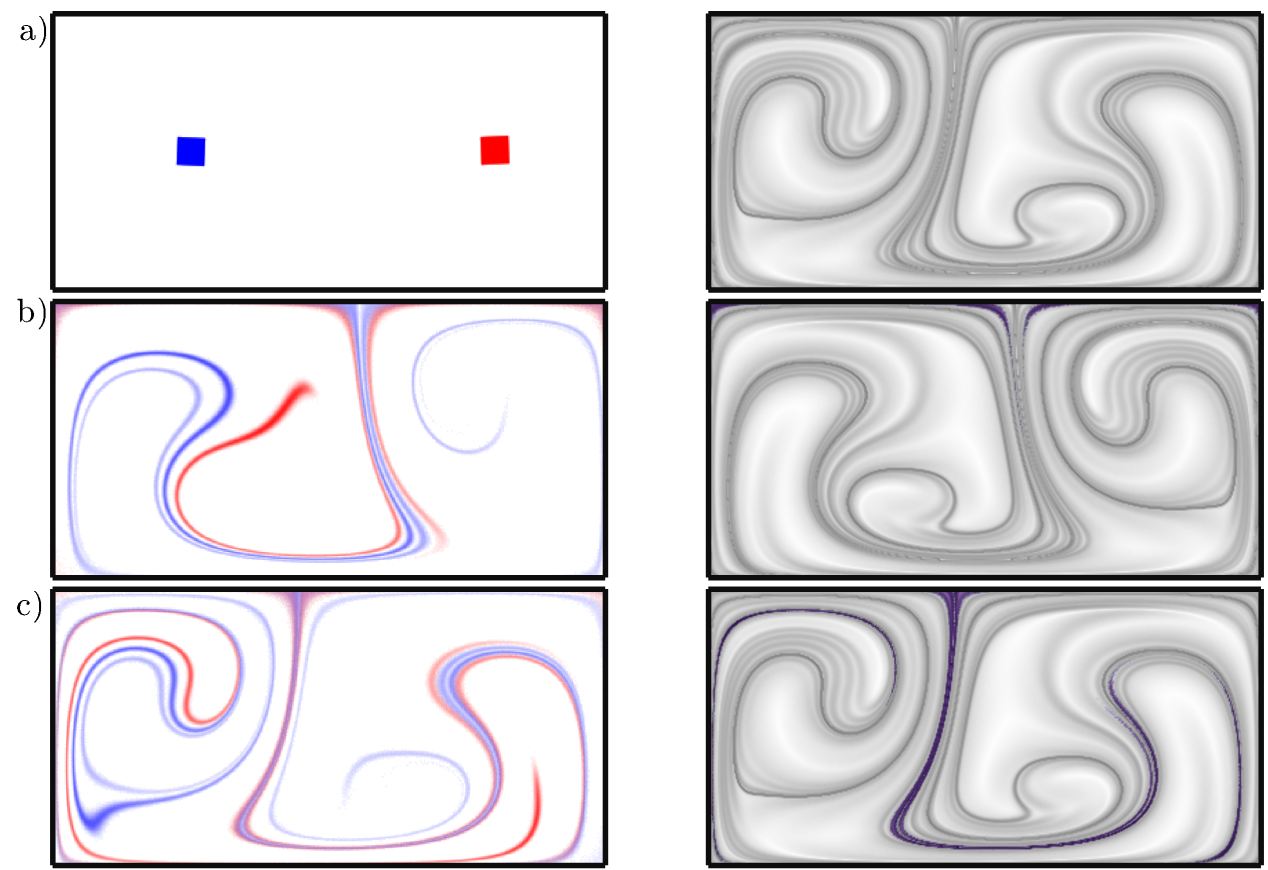

d)
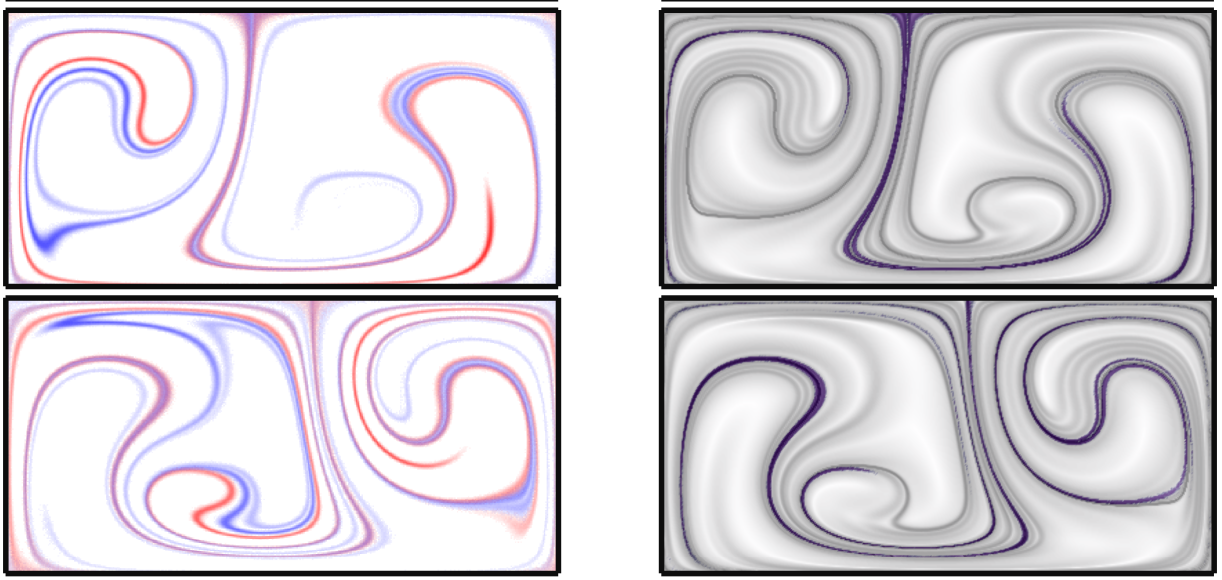

FIG. 7. Scalar concentrations (left) and reaction rates superimposed on the corresponding FTLE field (right) for the double gyre at a) $t=0$, b) $t=2.5 T_{0}$, c) $t=3 T_{0}$, and d) $t=3.5 T_{0}$. Note that the flow phase alternates between rows. The blue dye is initially centered at $(x, y)=(0.5,0.5)$ and red dye at $(1.6,0.5)$.

\section{Taylor Vortices}

Representative scalar concentrations and corresponding reaction rates for this flow are shown at four different times in Fig. 8. Even for this more complex flow, stirring of the two initially distant scalars leads to coalescence and initiation of reactions on the LCS.

\section{B. Reaction Rate Enhancement}

The results of the previous section show qualitatively that Lagrangian coherent structures serve as spatial templates for reactions in a range of $2 \mathrm{D}$ flows. We now turn to a quantitative investigation of the reactions rates. Because Crimaldi and Kawakami ${ }^{10}$ previously reported 


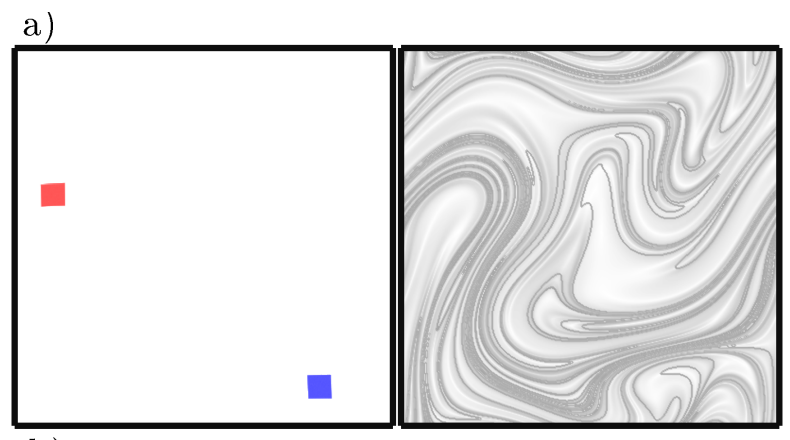

b)

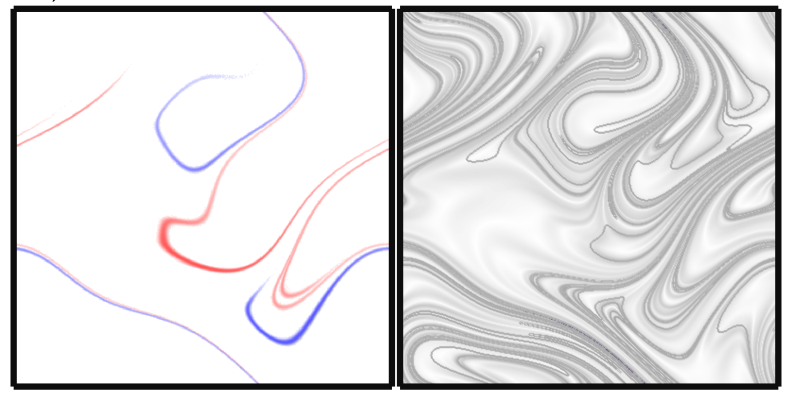

c)

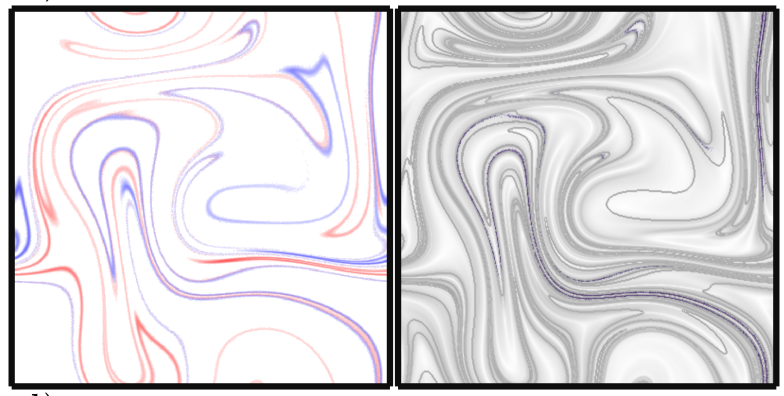

d)

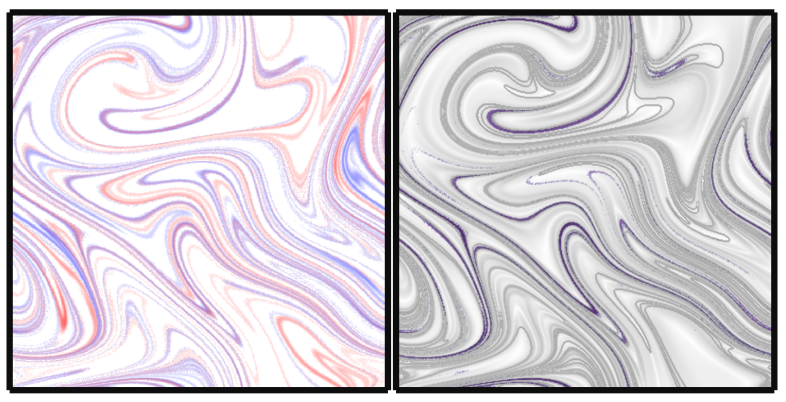

FIG. 8. (left) Scalar concentrations (red and blue) and (right) reaction rates (purple) superimposed on the corresponding FTLE field (gray) for the Taylor vortex flow at a) $\left.t^{*}=0, \mathrm{~b}\right) t^{*}=2.25, \mathrm{c}$ ) $t^{*}=3.38$, and d) $t^{*}=4.51$, where $t^{*}=t 2 \pi / e A$ is the time scaled by the turnover time at the core radius $r_{0}$.

reaction rates for the cylinder wake case, we limit ourselves herein to the double gyre and Taylor vortex flow. For simplicity, and because we are interested fundamentally in the role 
of structured stirring in promoting reactant coalescence to enable reactions, we calculate reactions only in the low-Damköhler number limit (see App. A), when reaction timescales are sufficiently slow relative to advective timescales that the reactions do not measurably deplete the associated reactants. ${ }^{9}$ Because both the double gyre and Taylor vortex flows have bounded domains, it is convenient to normalize the reaction rates using the steady-state rate

$$
R_{s s}=k C_{1, s s} C_{2, s s}
$$

where $C_{i, s s}$ is the spatially constant, well-mixed concentration as $t \rightarrow \infty$, i.e., $C_{i, s s}=$ $\frac{1}{A_{D}} \int C_{i}(x, 0) d A$, where $A_{D}$ is the area of the full domain. The reaction rate enhancement factor is then defined to be the ratio of the instantaneous, spatially averaged reaction rate to $(3)$ :

$$
R^{*}(t)=\frac{1}{R_{s s} A_{D}} \int R(x, t) d A
$$

To compute the reaction rate enhancement in our models, we begin with a pair of point sources placed randomly in the domain. As is natural for a chaotic systems, $R^{*}(t)$ is highly sensitive to the initial locations of the sources. Representative values of $R^{*}$ as a function of time are shown in Fig. 9(a) for the double gyre, and in Fig. 9(b) for the Taylor vortex flow. In each case, since the scalars are separated at $t=0, R^{*}(0)=0$, and since the diffusivity is non-zero, $R^{*}(t) \rightarrow 1$ as $t \rightarrow \infty$. However, there are two qualitatively different types of behavior. For some initial conditions, $R^{*}$ rises monotonically to unity, while for others, $R^{*}$ may sometimes greatly exceed unity at intermediate times. An example of each behavior is shown for both flows in Fig. 9. In all cases, there is an initial time during which $R^{*}$ remains effectively zero while the scalars are being stretched and folded, but have not yet substantially overlapped. Depending upon the initial locations of the scalars, the concentrated filaments of the two scalars may coalesce on an FTLE ridge at a relatively early time; in this situation, $R^{*}$ rises to a peak before decaying back to unity. The magnitude of the peak depends on the rapidity of the coalescence (and therefore on the residual concentration). In cases with no reaction enhancement, the coalescence of filaments took longer to occur, and thus the concentrations on the filaments is significantly diluted. Indeed, the exponential stretching characteristic of a chaotic flow also reduces concentrations exponentially.

Because the behavior and magnitude of $R^{*}$ is sensitive to initial conditions, it is convenient to use a Monte Carlo approach to quantify the likelihood of observing reaction enhancement. To do this, we vary the initial location of the point sources and determine the peak value 

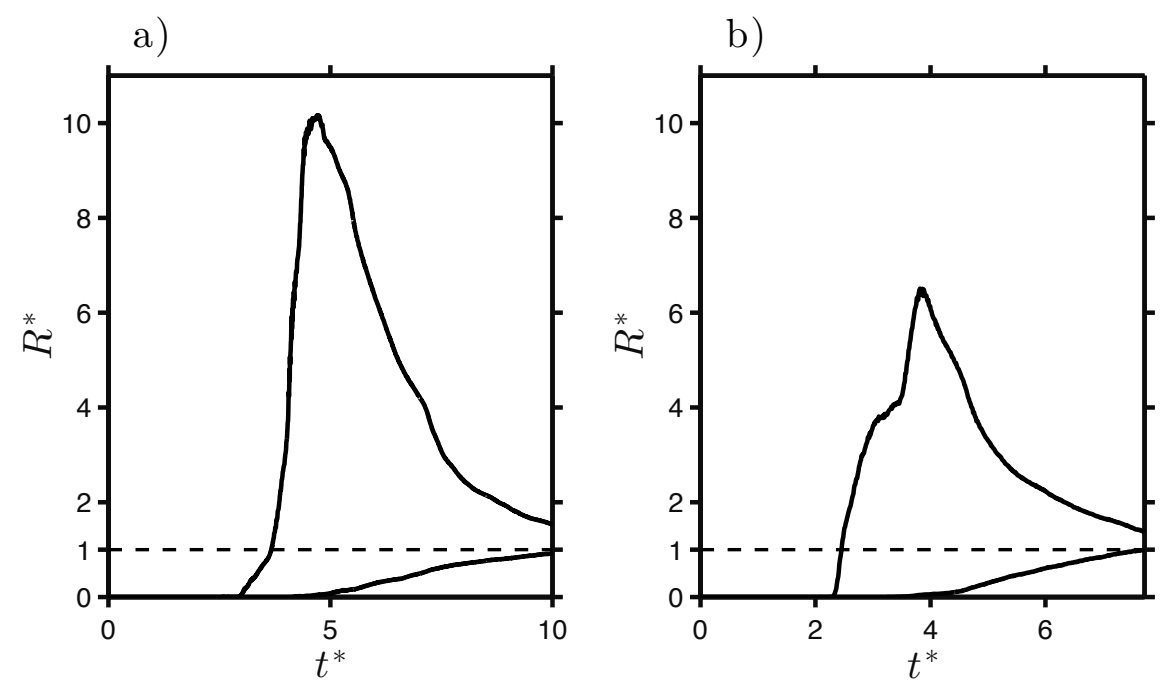

FIG. 9. Representative values of $R^{*}$ as a function of time for (a) the double gyre and (b) Taylor vortex flows, each shown for two initial conditions. For these simulations $L_{B}=0.01$ for (a) and $L_{B}=.07$ for (b), and the dye was initialized to a square with sized $L_{B}$. For the double gyre, time is nondimensionalized by the oscillation period $T_{0}$. For the Taylor vortex flow, time is nondimensionalized by $2 \pi / e A$, the turnover timescale at the core radius $r_{0}$.

of $R^{*}$ during each simulation. Figure 10 shows a cumulative density function (CDF) of maximum $R^{*}$ values from 5000 simulations with randomly chosen initial conditions. The $\mathrm{CDF}$ gives the probability that a given initial condition results in a maximum reaction rate greater than a chosen $R^{*}$. For example, about $3 \%$ of the trajectories give $R_{\max }^{*} \geq 10$. The data are not normally distributed and contain a fat, algebraic tail. The dashed black line in Fig. 10 corresponds to a power-law fit to the PDF,

$$
P\left(R_{\max }^{*}=x\right) \sim x^{-\beta}, \quad \beta=1.9,
$$

obtained from a Smirnov goodness-of-fit test. ${ }^{26}$ Note that this power law tail implies that the mean and variance of the PDF do not exist. Though a majority of the simulations do not result in significant enhanced reaction, about $65 \%$ of them had $R_{\max }^{*}>1$. Reaction enhancement, therefore, is a significant feature of flows with attracting Lagrangian coherent structures. 


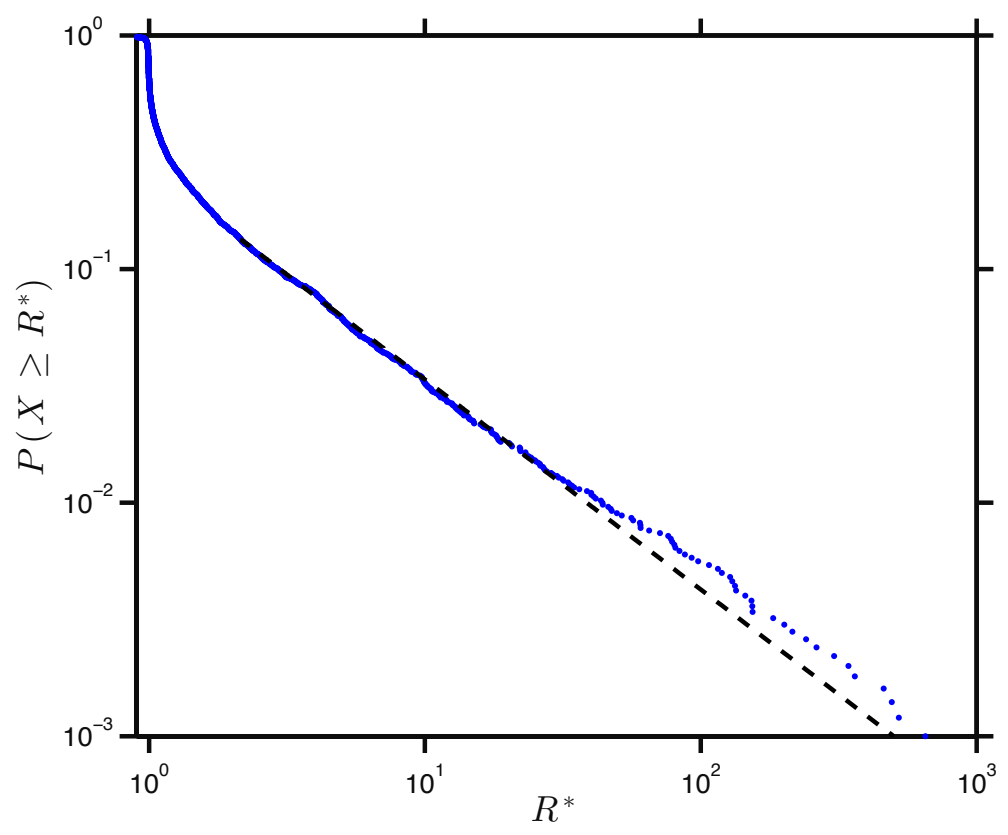

FIG. 10. Cumulative density function (dots) of maximum $R^{*}$ for 5000 simulations of the double gyre flow with random initial scalar locations. The dashed line shows the power-law fit (5).

\section{AN ANALYTICAL MODEL}

We now proceed to use simple scaling arguments to develop an analytical model that explains the large range of reaction enhancements seen in Fig. 10. From Figs. 6-8, it is clear that large reaction rates occur due to coalescence of concentrated scalar filaments on the LCS. However, a hallmark of chaotic flows is rapid dilution of diffusive scalar concentrations. Thus, the same structured stirring that produces filament coalescence also promotes dilution of filament concentrations. In order for reaction enhancement $\left(R^{*}>1\right)$ to occur, scalars must coalesce at an early enough time so that their concentrations are still sufficiently strong.

If diffusion is neglected, an initially localized passive scalar in a two-dimensional flow will stretch into a filament aligned in the direction of the local strain. When the flow is incompressible, there must be a corresponding contraction in a transverse direction such that area is preserved and the concentration remains constant. For diffusive scalars, the diffusive flux driven by strong transverse gradients eventually balances the stretching-induced contraction. Batchelor ${ }^{27}$ used scaling arguments to demonstrate that for turbulent flows this 


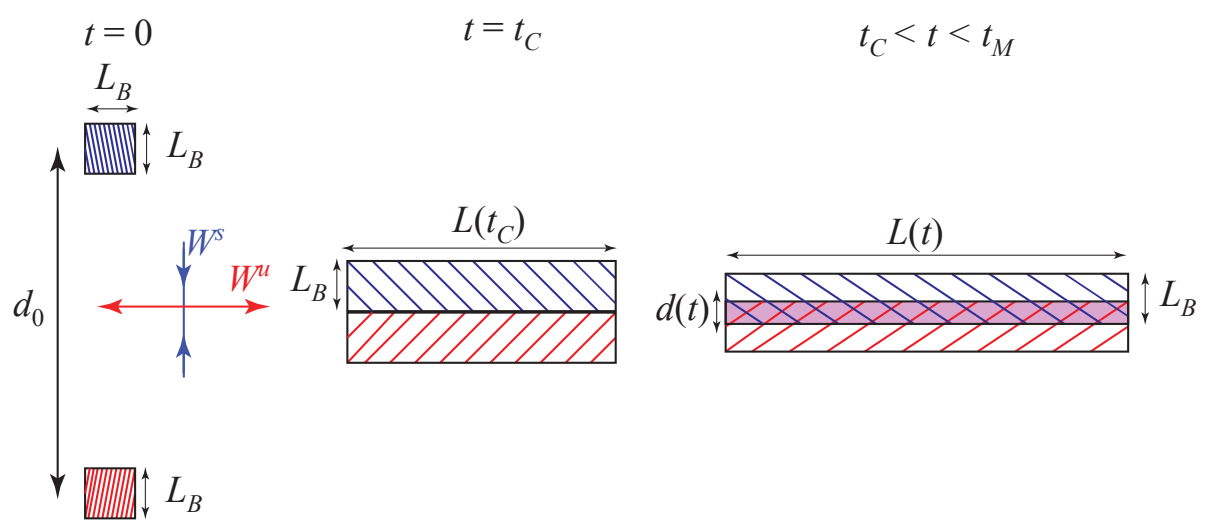

FIG. 11. Schematic for the analytical model at three times showing two scalars (red and blue) that start at $t=0$ a distance $d_{0}$ apart along the stable direction $W^{s}$, coalesce at $t=t_{C}$, stretching along the unstable direction $W^{u}$, and subsequently overlap on an area $A_{o}$ (purple) for $t>t_{C}$.

equilibrium filament width (now known as the Batchelor scale) is

$$
L_{B} \sim \sqrt{D \tau_{\eta}}
$$

where $D$ is the scalar diffusivity and $\tau_{\eta}$ is the Kolmogorov time. When the flow is chaotic, $L_{B}$ is commonly estimated by replacing $\tau_{\eta}$ by $\lambda^{-1}$, where $\lambda$ is the largest Lyapunov exponent, a metric of the mean strain rate ${ }^{28}$ thus,

$$
L_{B} \sim \sqrt{\frac{D}{\lambda}} .
$$

A chaotic flow will cause a filament to stretch in the unstable direction with an exponentially growing length: on average, $L(t) \sim \exp (\lambda t)$. It simultaneously contracts along the stable direction, and so once it reaches the equilibrium width $L_{B}$, the concentration must decay exponentially as $\exp (-\lambda t)$ (ignoring any reactions).

As a simple model, we consider two scalars with the same initial concentration, $C_{0}$, localized to squares of size $L_{B} \times L_{B}$ (Fig. 11). We will suppose, as the simplest model, that the flow is uniformly hyperbolic with a positive Lyapunov exponent $\lambda$, and that the stable and unstable directions are independent of the position of the Lagrangian element. Diffusion will maintain the width of the scalars in the stable direction at the Batchelor scale; however, they will stretch exponentially along the unstable direction - we assume that this holds even for macroscopic lengths, ignoring the folding that also must occur. Thus the area occupied 
by each scalar is $A(t)=L_{B}^{2} e^{\lambda t}$, and its concentration is

$$
C_{1}(t)=C_{2}(t)=C_{0} e^{-\lambda t}
$$

However, this exponential decay will stop when the exponential stretching causes the scalar to fill the entire domain, i.e., the area $A_{D}$. Thus the decay is valid up to a "mixing time" when $A\left(t_{M}\right)=A_{D}$ or to

$$
t_{M}=\frac{1}{\lambda} \log \left(\frac{A_{D}}{L_{B}^{2}}\right) .
$$

We will assume that the scalars are initially separated a distance $d_{0}$ along the stable manifold (nominally the vertical direction in Fig. 11), but they are at the same unstable location (i.e., there is no horizontal separation in Fig. 11). Given the exponential contraction in the stable direction, the stable distance will decrease exponentially with time

$$
d(t)=d_{0} e^{-\lambda t}
$$

When $d(t)=L_{B}$, the scalars come into contact; this is the "coalescence time",

$$
t_{C}=\frac{1}{\lambda} \log \left(\frac{d_{0}}{L_{B}}\right)
$$

and the reaction is initiated. As the vertical distance between the centers of the scalars continues to decrease according to Eq. (8), they overlap, i.e., the purple rectangle in Fig. 11. Generally, the overlap area is

$$
A_{o}(t)=\left\{\begin{array}{ll}
0, & 0<t<t_{C} \\
L_{B} e^{\lambda t}\left(L_{B}-d(t)\right), & t_{C}<t<t_{M} . \\
\frac{A_{D}}{L_{B}}\left(L_{B}-d(t)\right), & t_{M}<t
\end{array} .\right.
$$

The integrated reaction rate is thus $\int R(x, t) d A=k C_{1}(t) C_{2}(t) A_{o}(t)$. Since the steady state reaction rate, Eq. (3), is $R_{s s}=k\left(C_{0} L_{B}^{2} / A_{D}\right)^{2}$, then the normalized rate Eq. (4) becomes

$$
R^{*}(t)= \begin{cases}0, & 0<t<t_{C} \\ e^{\lambda\left(t_{M}-t\right)}\left(1-e^{-\lambda\left(t-t_{C}\right)}\right), & t_{C}<t<t_{M} . \\ 1-e^{-\lambda\left(t-t_{C}\right)}, & t_{M}<t\end{cases}
$$

This implies that $R^{*}(t)$ reaches a maximum at $t_{\text {max }}=t_{C}+\log 2 / \lambda$, provided that $t_{C} \leq$ $t_{\text {max }} \leq t_{M}$, with the peak value

$$
R_{\max }^{*}=\frac{1}{4} e^{\lambda\left(t_{M}-t_{C}\right)}=\frac{A_{D}}{2 L_{B}^{2}} e^{-\lambda t_{\max }}
$$




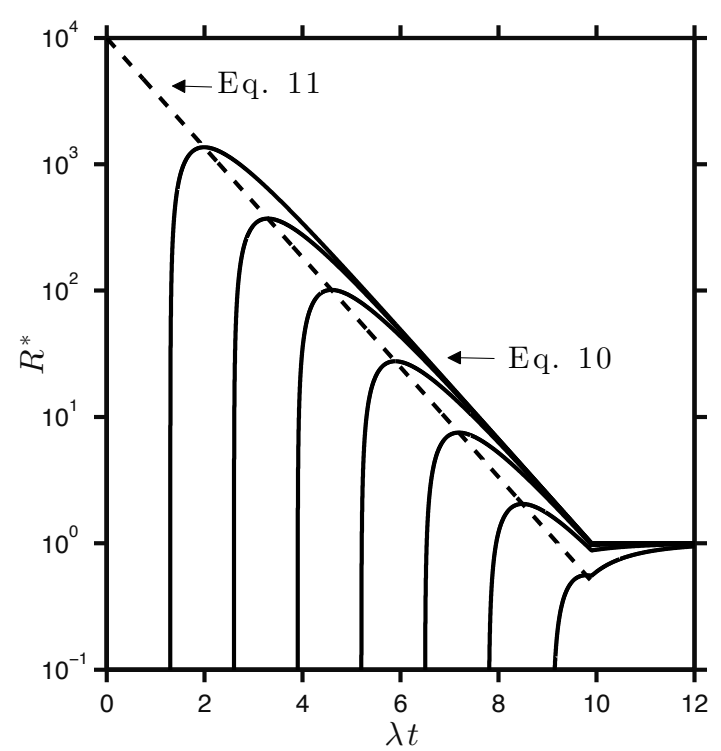

FIG. 12. A series of normalized reaction rate curves from (10) (solid lines) for various coalescence times, $t_{C}$, with $\lambda t_{M}=9.903$, corresponding to $A_{D} / L_{B}^{2}=2(10)^{4}$. The peak reaction rate (dashed line) is given by Eq. (11).

The enhancement is largest when the coalescence time is small, or equivalently when the initial separation distance $d_{0}$ approaches the Batchelor scale. The pre-factor $A_{D} / L_{B}^{2}$ in Eq. (11) represents the maximum possible enhancement for scalars that completely overlap at $t=0$. If $R^{*}(t)$ has a peak at some positive time, $t_{\max }>0$, then the initial scalars have at most $50 \%$ overlap, $d_{0} \geq \frac{1}{2} L_{B}$, and $R_{\max }^{*}$ is at most half of this theoretical maximum. Curves from Eq. (10) for seven values of $t_{C}$ are shown in Fig 12 (solid lines) for the case that $A_{D} / L_{B}^{2}=2(10)^{4}$. The peak value (Eq. (11)) is also shown in Fig. 12 (dashed line) as a function of $\lambda t$. A comparison of these curves with those in Fig. 9 shows that the competing effects of scalar coalescence and dilution, described by Eq. (10), give rise to the behavior that we observe in the simulations.

To test the validity of (10), we performed Monte Carlo simulations for both the double gyre and Taylor vortex flows. The scalars are given initially uniform concentrations on squares of size $L_{B} \times L_{B}$, with the diffusivity chosen so that $A_{D} / L_{B}^{2}=2(10)^{4}$. The scalars were placed along the repelling LCS in order to avoid elliptic islands in the flow. Initial overlap of the two squares was permitted (this corresponds to a negative coalescence time); however, in the vast majority of the initial conditions there was no overlap. For each simulation, the 
peak $R^{*}$ value and the corresponding time $t_{\max }$ are noted. Results are shown in Fig. 13(a) for the double gyre and in Fig. 13(b) for the Taylor vortex. To compare with the analytical model, $\lambda$ is estimated to be spatial mean of the FTLE; i.e., $\lambda=0.13$ for the double gyre and $\lambda=0.32$ for the Taylor vortex. The $R^{*}$ values from the Monte Carlo data generally fall below the bound given by (11), though there are a few points above, especially for larger values of $\lambda t_{\max }$. This is due, we believe, to scalars not being stretched at the maximal, idealized rate of the model. Indeed, the flows shown in Figs. 7-8 are certainly not uniformly hyperbolic, and their unstable manifolds fold in a complex way. This could give rise to concentrations larger than predicted by the model, since diffusive dilution is a much slower process than stretching induced dilution.

The large spread in $R^{*}$ values (across four orders of magnitude) seen in Fig. 13 for small $\lambda t_{\max }$ is probably due a variation in the filament overlap that can occur at these early times. In particular there there is no reason that the randomly placed scalars should be "vertically" aligned like we have assumed in the idealized model. In other words, they can approach the same unstable manifold from "different" stable manifolds - and therefore have lateral separation that reduces the overlap area. This spread in enhancement values is reduced for larger times since the magnitude of reaction enhancement decays at an exponential rate, as predicted by (11).

\section{DISCUSSION}

Lagrangian coherent structures have been demonstrated to play a crucial role in bringing distant scalars together. This idea has a wide range of applicability, ranging from the biological sciences to combustion chemistry. This paper has shown that LCS can serve as templates for reaction zones and that the peak reaction rate enhancement appears to obey a universal, power-law distribution. This contrasts with the predominant viewpoint in the existing literature that maximum reaction rates occur when the system reaches a well-mixed state. Under this latter view, optimizing reactions corresponds to maximizing mixing in the sense of rapid and uniform spread of Lagrangian scalars over the flow domain. However, our work has shown that coherent structures in fluid flows can result in enhanced reaction at intermediate time scales and that the peak can be orders of magnitude greater than the steady state rate. The mechanism responsible for this is the persistent straining 

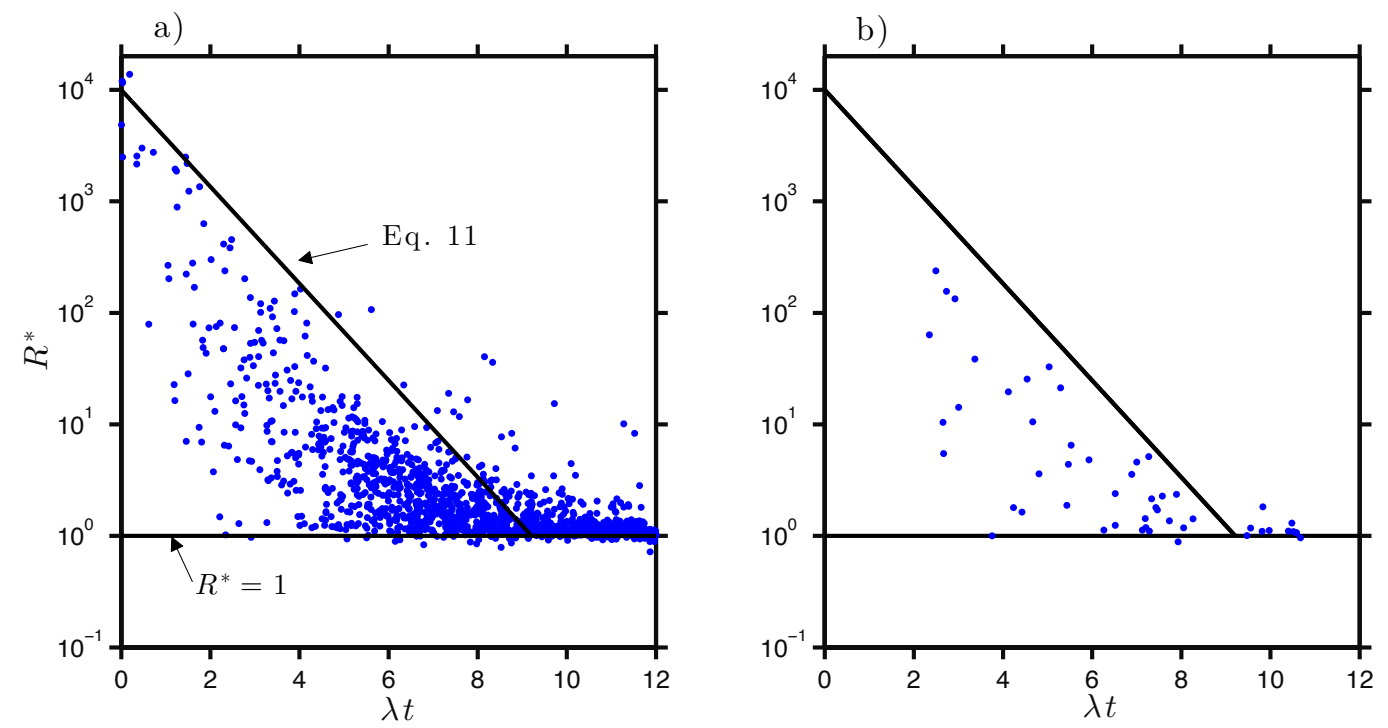

FIG. 13. Peak reaction $\left(R^{*}\right)$ and peak reaction times from double gyre (a)) and Taylor vortex (b)) from Monte Carlo simulations. The solid line shows (11) for $A_{D}=2, L_{B}=0.01$ with $\lambda=0.13$ for the double gyre, and $A_{D}=100, L_{B}=0.0707$ with $\lambda=0.32$ for the Taylor vortex.

of an attracting LCS that causes the contraction of the scalars onto filaments where the concentrations can be much larger than in a well-mixed system.

\section{ACKNOWLEDGMENTS}

This work was supported by the National Science Foundation (NSF) under grants PHY1205816 to JPC and DMS-1211350 to JDM. This work utilized the Janus supercomputer, which is supported by the NSF award CNS-0821794 and the University of Colorado Boulder. The Janus supercomputer is a joint effort of the University of Colorado Boulder, the University of Colorado Denver and the National Center for Atmospheric Research

\section{Appendix A: Damköhler Number and the Integration Time for FTLE}

The results in Sec. III show that LCS do indeed serve as templates for the enhanced reaction zones within chaotic/turbulent flows, regardless of initial scalar placement. Reactions are initiated when the scalars coalesce on the LCS and are then transported throughout the flow. As LCS begin to dissipate, however, it is possible that reactants that coalesced many turnover times ago will have not yet completely reacted. Such remnant reaction zones will 
no longer appear to be on the FTLE ridge. In such a slowly reacting system, the FTLE field needs to to be integrated for a time period appropriate to the reaction rate. Longer integration times will lengthen the approximate unstable manifolds and give a template for reaction zones over a timescale that can match that of the reaction. By contrast, in a highly reactive system, a shorter FTLE integration time is sufficient to capture the reaction zones. How long one grows the manifold, therefore, is entirely dependent upon the reactivity of the system.

Reactivity is quantified by the advective Damköhler number, which compares the advective timescale, $t_{a}$ of the flow to the reaction timescale, $t_{r}$, of the scalars.

$$
D a=\frac{t_{a}}{t_{r}}
$$

Using this definition, the Damköhler number becomes

$$
D a=\frac{t_{a} k M}{L^{2}}
$$

where $k$ is the reaction rate constant, $M$ is the total mass of one of the scalars, and $L$ is the initial separation distance between the two scalars.

By using this scaling, a $D a \ll 1$ indicates that reactants are not being degraded appreciably, $D a \sim 1$ signifies that reactants will be depleted within a few turnover times of the flow, and $D a \gg 1$ indicates significant depletion of reactants without appreciable advection. The choice of integration time, $T$, to compute the FTLE field should therefore match the reactive timescale; that is,

$$
T \sim \frac{L^{2}}{k M}
$$

In any case, the flow will have appreciable effect on the reaction only when $D a \lesssim 1$.

In this study, we focused on the moment of coalescence and not on reaction kinetics; thus we set $D a=0$ for all simulations: the scalars were not degraded, but only advected and diffused. The integration time $T \sim 2-2.5$ turnover times, was chosen to resolve only the coalescence events.

\section{REFERENCES}

${ }^{1}$ E.R. Abraham. The generation of plankton patchiness by turbulent stirring. Nature, 391:577-580, 1998 . 
${ }^{2}$ N. Peters. Turbulent Combustion. Cambridge: Cambridge University Press, 2000.

${ }^{3}$ J.M. Ottino. Mixing, Chaotic Advection, and Turbulence. Annu. Rev. Fluid Mech., 22:207253, 1990.

${ }^{4}$ H. Aref. Stirring by chaotic advection. J. Fluid Mech., 143:1-21, 1984.

${ }^{5}$ F.J. Muzzio and M. Liu. Chemical reactions in chaotic flows. Chem. Eng. J., 64:117-127, 1996.

${ }^{6}$ G. Károlyi. Effective dimensions and chemical reactions in fluid flows. Phys. Rev. E, 76:046315, 2007.

${ }^{7}$ J.P. Crimaldi and R.K. Zimmer. The physics of broadcast spawning in benthic invertebrates. Annu. Rev. Mar. Sci., 6:141-165, 2014.

${ }^{8}$ J.P. Crimaldi and Browning H.S. A proposed mechanism for turbulent enhancement of broadcast spawning efficiency. J. Mar. Syst., 49:3-18, 2004.

${ }^{9}$ J.P. Crimaldi, J.R. Cadwell, and J.B. Weiss. Reaction enhancement of isolated scalars by vortex stirring. Phys. Fluids, 20(7):073605, 2008.

${ }^{10}$ J.P Crimaldi and T.R. Kawakami. Reaction of initially distant scalars in a cylinder wake. Phys. Fluids, 25:053604, 2013.

${ }^{11}$ M. Soltys and J. Crimaldi. Joint Probabilities and Mixing of Isolated Scalars Emitted from Parallel Jets. J. Fluid Mech., In Revision, 2014.

${ }^{12}$ P. Holmes, Lumley J.L., G. Berkooz, and Rowley C.W. Coherent Structures, Dynamical Systems and Symmetry. Cambridge: Cambridge University Press, 2012.

${ }^{13}$ G. Haller and G. Yuan. Lagrangian coherent structures and mixing in two-dimensional turbulence. Physica D, 147(3-4):352-370, 2000.

${ }^{14}$ T. Peacock and J. Dabiri. Introduction to Focus Issue: Lagangian Coherent Structures. Chaos, 20:017501, 2010.

${ }^{15}$ M. Farazmand and G. Haller. Computing Lagrangian Coherent Structures from their variational theory. Chaos, 22:013128, 2012.

${ }^{16}$ T. Peacock and Haller G. Lagrangian Coherent Structures: The hidden skeleton of fluid flows. Physics Today, 66(2):41-46, 2013.

${ }^{17}$ F. Lekien, C. Coulliette, A.J. Mariano, E.H. Ryan, L.K. Shay, G. Haller, and J. Marsden. Pollution release tied to invariant manifolds: A case study for the coast of Florida. Physica D, 210:1-20, 2005.

${ }^{18}$ C.S. Harrison, D.A. Siegel, and S. Mitarai. Filamentation and eddy-eddy interactions in 
marine larval accumulation and transport. Mar Ecol Prog Ser, 472:27-44, 2013.

${ }^{19}$ E. Tew Kai, V. Rossi, J. Sudre, H. Weimerskirch, C. Lopez, E. Hernandez-Garcia, F. Marsac, and V. Garçon. Top marine predators track Lagrangian coherent structures. Proc. Nat. Acad. Sci., 106(20):8245-8250, 2009.

${ }^{20}$ S.C. Shadden, F. Lekien, and J.E. Marsden. Definition and properties of Lagrangian coherent structures from finite-time Lyapunov exponents in two-dimensional aperiodicflows. Physica D, 212(3-4):271-304, 2005.

${ }^{21}$ T.S. Tél, G. Károlyi, A. Péntek, Z. 5 Toroczkai, C. Grebogi, and J. Kadtke. Chaotic advection, diffusion, and reactions in open flows. Chaos, 10:89-98, 2000.

${ }^{22}$ A.E. Perry, M.S. Chong, and T.T. Lim. The vortex-shedding process behind twodimensional bluff bodies. J. Fluid Mech., 116:77-90, 1982.

${ }^{23}$ D.F. Carlson, E. Fredj, Hezi G., and V. Rom-Kedar. Deducing an upper bound to the horizontal eddy diffusivity using a stochastic Lagrangian model. Envrion. Fluid Mech., 10:499-520, 2010.

${ }^{24}$ H. Aref. Point vortex dynamics: A classical mathematics playground. J. Math. Phys., 48:065401, 2007.

${ }^{25}$ R.L. Panton. Incompressible Flow. John Wiley Sons, Inc, 1996.

${ }^{26}$ A. Clauset, C.R. Shalizi, and M.E.J. Newman. Power law distributions in empirical data. SIAM Review, 51:661-703, 2009.

${ }^{27}$ G.K. Batchelor. Small-scale variation of convected quantities like temperature in turbulent fluid. Fluid Mechanics, 5(1):113-133, 1959.

${ }^{28}$ H. Aref, J.R. Blake, M. Budišić, J.H.E. Cartwright, H.J.H. Clercx, U. Feudel, R. Golestanian, E. Gouillart, Y.L. Guer, G.F. van Heijst, et al. Frontiers of chaotic advection. arXiv preprint arXiv:1403.2953, 2014. 